\title{
Healthcare worker influenza vaccination and sickness absence - an ecological study
}

\author{
Authors: Miguel Pereira, ${ }^{\mathrm{A}}$ Siân Williams, ${ }^{\mathrm{B}}$ Louise Restrick, ${ }^{\mathrm{C}}$ Paul Cullinan ${ }^{\mathrm{D}}$ and Nicholas S Hopkinson ${ }^{\mathrm{E}}$ on behalf of \\ the London Respiratory Network
}

\begin{abstract}
Although Influenza vaccination is recommended for healthcare workers, vaccination rates in UK healthcare workers are only around $50 \%$. We investigated the association between NHS sickness absence rates (using data from Health and Social Care Information Centre quarterly reports), staff vaccination rates and influenza vaccine efficacy (from Public Health England), influenza deaths (from the Office of National Statistics) and staff satisfaction (from www.NHSstaffsurveys. com). Data from 223 healthcare trusts covered approximately 800,000 staff in each of four influenza seasons from 2011; overall staff sickness rate was roughly $4.5 \%$. Annual vaccination rates varied between $44 \%$ and $54 \%$. Higher NHS trust vaccination rates were associated with reduced sickness absence $(\beta=-0.425$ [95\% CI -0.658 to -0.192$], p<0.001)$. Thus, a $10 \%$ increase in vaccination rate would be associated with a $10 \%$ fall in sickness absence rate. Influenza vaccination for NHS staff is associated with reduced sickness absence rates.
\end{abstract}

KEYWORDS: Healthcare worker, influenza, occupational health, vaccination

\section{Introduction}

Annual vaccination against influenza is recommended for all UK NHS staff to reduce transmission to patients and help prevent sickness absence, thereby reducing strain on services at times of peak demand. ${ }^{1,2}$ During a mild influenza season around $23 \%$ of healthcare workers may become infected with flu; $28-59 \%$ of these have subclinical, though potentially transmissible, illness. ${ }^{3,4}$ Healthcare workers may therefore represent an important vector for transmission to patients and studies in long-term care have found that immunising healthcare personnel against influenza each year decreases all-cause

\footnotetext{
Authors: ${ }^{\text {A }}$ research fellow, National Heart and Lung Institute, London, UK; B programme manager, London Respiratory Network, London, UK; ' Consultant chest physician, London Respiratory Network and Whittington Health, London, UK; ${ }^{D}$ professor of occupational and environmental respiratory disease, National Heart and Lung Institute, London, UK; ${ }^{E}$ reader in respiratory medicine, London Respiratory Network and NIHR Respiratory Biomedical Research Unit, Royal Brompton and Harefield NHS Foundation Trust and Imperial College London, UK
}

mortality among residents. ${ }^{5-9}$ Any vaccine hesitancy may also affect their intention to recommend vaccination to patients and therefore patients' vaccine uptake, ${ }^{10}$ which is of particular concern given the survival benefits associated with influenza vaccination in older people and those with chronic illness. ${ }^{11}$ However, influenza vaccine uptake by NHS staff remains relatively low, at around half of eligible staff. ${ }^{1}$ Survey data comparing the attitudes of staff who had or had not been vaccinated in the preceding year suggests that staff who had not had the influenza vaccination were more likely to respond that it was too much trouble to get the vaccination, that they felt they were not at risk of getting influenza and that they thought the vaccination would make them feel unwell. ${ }^{12,13}$ US healthcare worker influenza vaccination rates are also persistently low. ${ }^{14}$ Additional evidence that influenza vaccination has a positive effect on healthcare workers' own wellbeing might further influence NHS staff beliefs and behaviours with respect to being vaccinated. We therefore aimed to use routinely collected data to examine the hypothesis that NHS staff influenza vaccination rates would be associated with reduced sickness absence. We adjusted this for the severity of the influenza season (using influenza mortality), for vaccine efficacy and for NHS staff satisfaction survey findings, which might also influence staff sickness behaviour.

\section{Methods}

\section{Definition of sickness absence rate}

We studied sickness absence rates in NHS trusts in England for 2011-12, 2012-13, 2013-14 and 2014-15. These are reported quarterly by the Health and Social Care Information Centre and are freely available online. Sickness absence is calculated from the electronic staff record and is defined as the ratio between the 'full time equivalent (FTE) number of days sick' and 'FTE number of days available'. We obtained the monthly rates for all NHS trusts in England for the seasons 2011-12, 2012-13, 2013-14 and 2014-15 and calculated the overall sickness absence rate for the influenza season, which we defined as the period between October and March.

Ethical approval was not required for this study.

\section{Independent variables and dataset compilation}

Our primary independent variable was seasonal influenza vaccine uptake in healthcare workers. These data are released 
by Public Health England and are publicly available on their website. Annual figures for seasonal influenza vaccine uptake (September to August) are available for each NHS trust with a breakdown by type of healthcare worker (HCW): doctors, nurses, other clinical staff and non-clinical support staff. As for the sickness absence data, we obtained reports for the seasons 2011-12, 2012-13, 2013-14 and 2014-15.

To adjust for possible confounding, we considered other variables that could be associated with seasonal influenza vaccine uptake, influenza severity and NHS staff sickness absence. In particular, we retrieved data regarding influenza vaccine efficacy, influenza deaths and NHS staff satisfaction with their institution for each of the influenza seasons.

Influenza vaccine efficacy estimates are available from Public Health England as part of the annual flu programme. We obtained the overall estimates of the influenza vaccine efficacy for influenza A and B for each of the four seasons in our study.

We obtained influenza deaths data from the Office National Statistics (www.ons.gov.uk), which reports the number of deaths in England by geographic region, sex, age group and cause of death. We selected the deaths caused, specifically, by influenza (ICD-10 codes J09-J11). The death registries per cause of death are aggregated by year and we considered the deaths due to influenza for the full years 2012, 2013, 2014 and 2015, which we directly matched with the flu seasons we analysed.

We included data regarding NHS staff satisfaction (www.nhsstaffsurveys.com) because this could influence sickness absence and influenza vaccine uptake - both might be better in organisations with higher levels of staff engagement.

\section{Statistical analysis}

Dataset compilation and all statistical analyses were carried out using STATA version 13.0 (StataCorp). A linear mixedeffects model, with a random effect for NHS trust, was used to evaluate the effect of NHS staff influenza vaccine uptake and sickness absence during the 'influenza season' months (October to March). Association analysis was performed for influenza vaccine uptake in all HCWs and for each staff group individually. In the latter, we applied the Benjamini-Hochberg method to correct for multiple testing as we fitted one regression model per staff group. The following covariates were included in our analysis: influenza vaccine efficacy, flu deaths and the NHS staff 'overall engagement score' as a measure of staff satisfaction with the organisation. These are expected to be related to both the sickness absence and influenza vaccine uptake and act as potential confounders. Statistical significance was determined by considering a type I error probability below $5 \%(\alpha<0.05)$. As a sensitivity analysis, we repeated all association testing for the 'non-influenza season' months, April to September, with the purpose of assessing if the effects observed were specific to the influenza season.

Although this is an ecological study, all analyses and data reporting were performed according to the STrengthening the Reporting of OBservational studies in Epidemiology (STROBE) statement whenever applicable. ${ }^{15}$

\section{Results}

223 NHS trusts (out of 245) were included in the study, comprising 17 acute (specialist) trusts, 131 acute trusts,

\begin{tabular}{|c|c|c|c|c|}
\hline \multirow[b]{3}{*}{ Total staff } & \multicolumn{4}{|c|}{ Flu season } \\
\hline & 2011-12 & $2012-13$ & 2013-14 & 2014-15 \\
\hline & 802,710 & 811,654 & 821,214 & 830,611 \\
\hline Doctors & $\begin{array}{l}95,251 \\
(11.9 \%)\end{array}$ & $\begin{array}{l}101,394 \\
(12.5 \%)\end{array}$ & $\begin{array}{l}101,639 \\
(12.4 \%)\end{array}$ & $\begin{array}{l}102,849 \\
(12.4 \%)\end{array}$ \\
\hline Nurses & $\begin{array}{l}314,652 \\
(39.2 \%)\end{array}$ & $\begin{array}{l}307,449 \\
(37.9 \%)\end{array}$ & $\begin{array}{l}315,622 \\
(38.4 \%)\end{array}$ & $\begin{array}{l}321,365 \\
(38.7 \%)\end{array}$ \\
\hline $\begin{array}{l}\text { Other clinical } \\
\text { staff }\end{array}$ & $\begin{array}{l}146,997 \\
(18.3 \%)\end{array}$ & $\begin{array}{l}152,121 \\
(18.7 \%)\end{array}$ & $\begin{array}{l}149,584 \\
(18.2 \%)\end{array}$ & $\begin{array}{l}148,939 \\
(17.9 \%)\end{array}$ \\
\hline $\begin{array}{l}\text { Non-clinical } \\
\text { support staff }\end{array}$ & $\begin{array}{l}245,810 \\
(30.6 \%)\end{array}$ & $\begin{array}{l}250,690 \\
(30.9 \%)\end{array}$ & $\begin{array}{l}254,369 \\
(31 \%)\end{array}$ & $\begin{array}{l}257,458 \\
(31.0 \%)\end{array}$ \\
\hline
\end{tabular}

10 ambulance trusts, 55 mental health/learning disability trusts, and 10 community trusts. After merging all the datasets, over 800,000 healthcare workers were included for each influenza season. Table 1 shows staff numbers within these trusts for the last four influenza seasons, broken down by staff role. The sample of healthcare workers was composed, on average, of approximately $12.4 \%$ doctors, $38.5 \%$ nurses, 18.4 other clinical support staff and $30.7 \%$ non-clinical support staff across the four seasons. The characteristics of the sample regarding the outcome and the covariates analysed are summarised in Table 2 . The overall sickness absence rate was approximately $4.5 \%$, with the highest rate of $4.67 \%$ being observed in the $2014-15$ season. Influenza vaccine uptake in all healthcare workers was roughly $44 \%$ in the $2011-12$ and $2012-13$ seasons and 54\% in the 2013-14 and 2014-15 seasons, which suggests an increase in influenza vaccine uptake.

Fig 1 represents the trends in influenza vaccine uptake across the three influenza seasons considered by breakdown by staff group. Overall, vaccine uptake remained constant between the 2011-12 and 2012-13 seasons and increased by approximately $10 \%$ in the 2013-14 season in all groups while remaining stable in the 2014-15 season. Healthcare workers other than doctors and nurses (ie 'Other clinical staff') and non-clinical support staff had higher rates of vaccine uptake, followed by doctors and then nurses, the last reaching a vaccination rate of approximately $48 \%$ in the 2013-14 and 2014-15 seasons (Table 2).

Table 3 shows the estimated coefficients, 95\% confidence intervals and p-values in the mixed-effects model, analysing the relationship between sickness absence rate and influenza vaccine uptake, where vaccine uptake was significantly associated with a reduction in sickness absence rate $(\beta=-0.425, p<0.001)$. In particular, an increase of $10 \%$ in influenza vaccine uptake, such as the one observed between the 2012-13 and 2013-14 influenza seasons, would be associated with a decrease in approximately 0.43 percentage points in the absolute sickness absence rate. Considering the average sickness absence rate was $4.5 \%$ across the four influenza seasons, this translates into a $10 \%$ relative decrease in the sickness rate, which suggests that increasing vaccine uptake can have a significant practical impact.

We found that influenza vaccine efficacy and influenza deaths are both significantly associated with NHS staff sickness absence. As expected, an increase in vaccine efficacy 
Table 2. General sample characteristics by flu season (2011-12, 2012-13, 2013-14, 2014-15) in NHS trusts in England $(n=223)$

\begin{tabular}{|c|c|c|c|c|c|c|c|c|}
\hline & \multicolumn{8}{|c|}{ Flu season } \\
\hline & \multicolumn{2}{|c|}{$2011-12$} & \multicolumn{2}{|c|}{ 2012-13 } & \multicolumn{2}{|c|}{ 2013-14 } & \multicolumn{2}{|c|}{$2014-15$} \\
\hline & Mean & SD & Mean & SD & Mean & SD & Mean & SD \\
\hline Sickness absence rate & 4.467 & 0.862 & 4.592 & 0.910 & 4.376 & 0.879 & 4.635 & 0.951 \\
\hline Flu vaccine uptake, \% & 43.6 & 15.3 & 44.0 & 16.8 & 54.3 & 15.8 & 54.3 & 15.6 \\
\hline Doctors & 45.1 & 22.0 & 43.4 & 22.3 & 53.8 & 21.0 & 53.2 & 23.2 \\
\hline Nurses & 38.5 & 17.1 & 39.0 & 18.0 & 48.0 & 18.1 & 47.7 & 17.4 \\
\hline Other clinical staff & 49.4 & 20.6 & 49.8 & 20.6 & 59.1 & 19.6 & 58.6 & 18.9 \\
\hline Non-clinical support staff & 49.4 & 23.1 & 50.6 & 24.8 & 60.1 & 23.0 & 60.9 & 22.7 \\
\hline
\end{tabular}

is associated with a reduction in sickness absence $(\beta=-0.008$, $\mathrm{p}<0.001)$ as the vaccine will confer more protection and reduce overall influenza infections. On the other hand, influenza deaths are associated with an increase in sickness absence ( $\beta=0.004, p<0.001)$, which is to be expected based on our underlying assumption that influenza deaths are a proxy for influenza severity in a given year.

The 'overall engagement score' ranged from 2.78 to 4.03 out of 5 in the 2015 NHS staff survey among the five the types of NHS trusts included in our study, with an average score of approximately 3.7 in the four surveys considered (years 2012, 2013, 2014 and 2015). This score was also significantly associated with a reduction in sickness absence rate $(\beta=-1.111$, $\mathrm{p}<0.001)$ and shows the largest effect of all the covariates analysed. However, its impact is not expected to be as great as a change in vaccine uptake. Considering the average overall engagement score of 3.7 and that the maximum possible score is 5 , improving staff satisfaction to achieve its maximum score, an unlikely possibility, would be associated with a decrease of $30 \%$ in overall sickness absence. The same effect can be achieved with an increase of $30 \%$ in vaccine uptake by going from $50 \%$ of HCWs vaccinated to $80 \%$.

In order to identify in which employee groups there were significant associations between influenza vaccine uptake and sickness absence rate, we performed the same regression

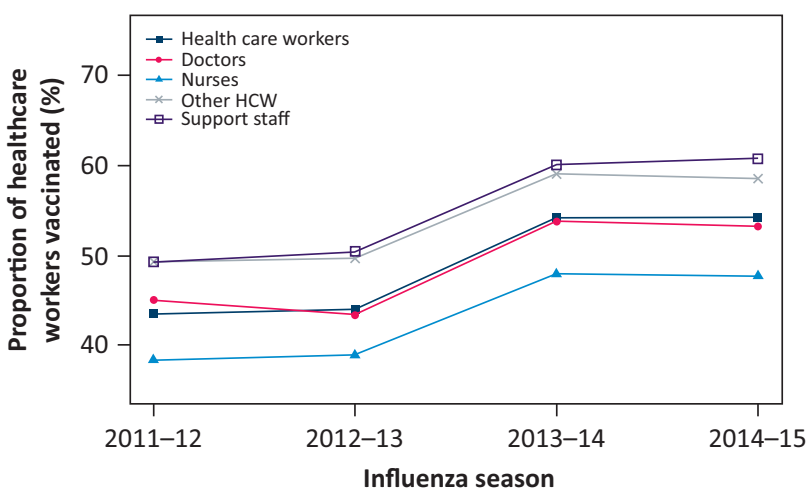

Fig 1. Trends in influenza vaccine uptake across the 2011-12, 2012-13, 2013-14 and 2014-15 influenza seasons. Results represented for each type of healthcare worker (HCW): doctors, nurses, other HCWs and nonclinical support staff. analysis for each individual class; the results are summarised in Table 4. We found a significant association between influenza vaccine uptake and sickness absence in all four employee groups analysed; this association remained significant after correcting for multiple testing by controlling for the false discovery rate using the Benjamini-Hochberg method. Nurses show a stronger association $(\beta=-0.378)$ than the other three groups, where $\beta=-0.220$. These results suggest that all employee groups are driving the association observed when we analyse all HCWs, with nurses being the main driver of this association.

As a sensitivity analysis, we repeated all regression analyses considering the sickness absence rates for NHS staff in the months of April to September, the 'non-influenza' seasons, in the years 2012 to 2015 . The purpose for this analysis was to address the hypothesis of a 'healthy user bias'. In this scenario, vaccinated staff should show lower sickness absence rates in both the influenza and non-influenza seasons. However, when all HCWs are considered (Table 5), we found a weak association in the opposite direction to that seen during the influenza season, with higher vaccination rates associated with slightly higher sickness absence rates $(\beta=0.212, \mathrm{p}<0.046)$. This effect

Table 3. Association between influenza vaccine uptake and sickness absence rate adjusted for flu vaccine efficacy, flu deaths in England, type of NHS trust and Overall Engagement Score obtained from the NHS staff survey

\begin{tabular}{|c|c|c|c|}
\hline & Beta & $95 \% \mathrm{CI}$ & p-value \\
\hline Influenza vaccine uptake & -0.425 & $\begin{array}{l}(-0.658 \text { to } \\
-0.192)\end{array}$ & $<0.001$ \\
\hline Influenza vaccine efficacy & -0.008 & $\begin{array}{l}(-0.010 \text { to } \\
-0.006)\end{array}$ & $<0.001$ \\
\hline Influenza deaths & 0.004 & $\begin{array}{l}(-0.001 \text { to } \\
0.000)\end{array}$ & $<0.001$ \\
\hline $\begin{array}{l}\text { Overall engagement score } \\
\text { (NHS staff survey) }\end{array}$ & -1.039 & $\begin{array}{l}(-1.399 \text { to } \\
-0.679)\end{array}$ & $<0.001$ \\
\hline Intercept & 9.004 & $\begin{array}{l}\text { (7.623 to } \\
10.384)\end{array}$ & $<0.001$ \\
\hline
\end{tabular}

Results correspond to a linear mixed-effects models with a random intercept for NHS trust 
Table 4. Association between influenza vaccine uptake in each class of healthcare worker and sickness absence rate

\begin{tabular}{|c|c|c|c|c|}
\hline & Beta & $95 \% \mathrm{CI}$ & $\mathrm{p}$-value & $\begin{array}{l}\text { Adjusted } \\
\text { p-value* }\end{array}$ \\
\hline Doctors & -0.218 & $(-0.385$ to -0.051$)$ & 0.011 & 0.014 \\
\hline Nurses & -0.378 & $(-0.598$ to -0.159$)$ & 0.001 & 0.004 \\
\hline $\begin{array}{l}\text { Other clinical } \\
\text { staff }\end{array}$ & -0.202 & $(-0.364$ to -0.039$)$ & 0.015 & 0.015 \\
\hline $\begin{array}{l}\text { Non-clinical } \\
\text { support staff }\end{array}$ & -0.232 & ( -0.381 to -0.083$)$ & 0.002 & 0.004 \\
\hline \multicolumn{5}{|c|}{$\begin{array}{l}\text { *p-values adjusted for multiple testing using the Benjamini-Hochberg method. } \\
\text { Results correspond to a linear mixed-effects model with a random intercept for } \\
\text { NHS trust for each class separately. Each model was adjusted for flu vaccine } \\
\text { efficacy, flu deaths in England, type of NHS trust and Overall Engagement } \\
\text { Score obtained from the NHS staff survey. }\end{array}$} \\
\hline
\end{tabular}

was only apparent among doctors (Table 6: $\beta=-0.175, \mathrm{p}<0.021$ ) when staff groups were analysed separately, but this association did not survive correction for multiple testing (adjusted p-value $=0.084)$. No association was detected between sickness absence and vaccine uptake during the 'non-influenza' seasons in the other staff groups (Table 6).

\section{Discussion}

The Council of The European Union has called for action to mitigate the impact of seasonal influenza by encouraging vaccination among healthcare workers. ${ }^{16}$ Supporting the validity of this approach, the principal finding of this study (an examination of administrative data collected from NHS trusts over a 4 -year period) was that higher influenza vaccination rates were associated with lower rates of staff sickness absence. A 10 percentage point increase in influenza vaccination rate was associated with approximately $10 \%$ fall in sickness absence rate.

This association does not prove causation, but there are credible reasons to believe that there is a causal link, given the expected impact of vaccination on influenza severity and transmission within the workplace and to and from household and other contacts.

Table 5. Sensitivity analysis - association between

influenza vaccine uptake in each class of healthcare worker and sickness absence rate in the months outside of the flu season (April to September)

\begin{tabular}{|c|c|c|c|}
\hline & Beta & $95 \% \mathrm{CI}$ & p-value \\
\hline $\begin{array}{l}\text { Influenza vaccine } \\
\text { uptake }\end{array}$ & 0.212 & (0.004 to 0.421$)$ & 0.046 \\
\hline $\begin{array}{l}\text { Influenza vaccine } \\
\text { efficacy }\end{array}$ & -0.003 & $(-0.005$ to -0.001$)$ & 0.001 \\
\hline Influenza deaths & 0.0002 & (0.0001 to 0.0004$)$ & 0.002 \\
\hline $\begin{array}{l}\text { Overall engagement } \\
\text { score (NHS staff survey) }\end{array}$ & -1.203 & $(-1.526$ to -0.879$)$ & $<0.001$ \\
\hline Intercept & 8.530 & (7.291 to 9.770 ) & $<0.001$ \\
\hline
\end{tabular}

\begin{tabular}{|c|c|c|c|c|}
\hline & Beta & $95 \%$ CI & p-value & $\begin{array}{l}\text { Adjusted } \\
\text { p-value* }\end{array}$ \\
\hline Doctors & 0.175 & $(0.026,0.324)$ & 0.021 & 0.084 \\
\hline Nurses & 0.040 & $(-0.157,0.237)$ & 0.689 & 0.925 \\
\hline $\begin{array}{l}\text { Other clinical } \\
\text { staff }\end{array}$ & 0.053 & $(-0.091,0.199)$ & 0.466 & 0.925 \\
\hline $\begin{array}{l}\text { Non-clinical } \\
\text { support staff }\end{array}$ & 0.001 & $(-0.127,0.083)$ & 0.925 & 0.925 \\
\hline
\end{tabular}

\section{Methodological issues}

A strength of the study is the availability of large administrative datasets covering a large majority of NHS staff in England. However, recording of vaccination status may not be complete if individuals have been vaccinated outside their workplace, for example in primary care. This is not likely to have been a source of systematic error. An important possible confounder is that organisations that are better organised might have higher levels of vaccination, but also have higher levels of staff satisfaction, with less time taken off work because of stress. However, we included results from the staff survey in our modelling and found that the relationship between vaccination rate and sickness absence was independent of staff satisfaction.

We do not have data available for staff use of neuramidase inhibitors, which might have had an impact on the duration of sickness absence in individuals with influenza. The roll out of UK childhood influenza immunisation might also have had some impact on sickness absence over time because of an overall reduction in transmission and reduction in absenteeism due to child illness. The use of agency staff, whose vaccination records are not incorporated in the data used here, may impact on the precision of our estimates although probably only to a small extent.

The ecological fallacy limits the inferences about the nature of individual behaviours that can be made based on the group to which those individuals belong. It is possible that some general confounding effect, linking influenza vaccination either to better overall health or to different sickness behaviour might be responsible for some of the association we observed. However, the sensitivity analysis makes this unlikely, as the effect of vaccination was only apparent during the vaccination season; at other times of the year, influenza vaccination rates were not associated with levels of sickness absence.

\section{Significance of findings}

In addition to evidence that staff vaccination protects vulnerable patients ${ }^{5-8}$ (with a pooled risk ratio across trials for all-cause mortality of 0.71 [95\% CI 0.59-0.85]), ${ }^{9}$ there are also some trial data already available to support a benefit to healthcare workers from vaccination. A randomised controlled trial of influenza vaccination in 547 workers in two paediatric hospitals found that, over 4 months of follow-up, vaccination 
reduced the number of days that staff felt unable to work because of sickness from 3.5 to 2.5 days. ${ }^{17}$ In a trial in 40 care homes for the elderly, HCW vaccination was associated with $42 \%$ lower sickness absence from work. ${ }^{7}$ It should be noted that systematic reviews of the data in this area have varied in their findings depending on methodology and the outcomes selected, with the strongest data available for all-cause mortality. ${ }^{18}$

Despite recommendations for all healthcare staff to be vaccinated, overall rates only ranged between $44 \%$ and $54 \%$. Several studies have investigated the reasons for this. In an online survey of 3,059 respondents working in the NHS, a positive predictor of having been vaccinated was the extent of agreement with the statement 'people working in healthcare should have the influenza vaccination every year' $(\mathrm{p}<0.001)$, with 'the influenza vaccination will make me unwell' $(\mathrm{p}<0.001)$ and 'the influenza vaccination was too much trouble for me' $(\mathrm{p}<0.001)$ retained as negative predictors. ${ }^{12}$

An online, anonymous survey of HCWs across five acute NHS trusts was undertaken in $2010 .{ }^{19}$ A total of 765 responses were obtained, of which the two main groups of participants were doctors of all grades (42\%) and qualified nurses (40\%). The willingness to be vaccinated increased with age. Senior doctors were the occupational group most likely to have been vaccinated, but where they did decline they mainly did so because they perceived influenza to be a minor illness. Females were more likely to decline vaccination because of a fear of side effects, whereas males (particularly younger ones), viewed influenza as a minor illness. Junior doctors cited lack of availability of immunisation sessions as one of the main reasons why they may not have had the vaccination.

The key impact of demonstrating that influenza vaccination has an effect on NHS staff sickness absence should be an increase in vaccination rates. There are several reasons for this. Firstly, although a major reason for the recommendation that HCWs should be vaccinated is to protect vulnerable patients, this altruistic reason can now be more confidently complemented by evidence of personal benefit to HCWs themselves. HCWs often share responsibilities for patient care within a team and may be motivated to reduce their risk of sickness as a way of minimising the burden on their colleagues. In addition, many HCWs cite practical difficulties with accessing influenza vaccination. An understanding by healthcare organisation leaders that a more effective influenza vaccination programme can reduce the impact of staff sickness on rotas, including the need to employ agency staff, should be an incentive to deliver a more comprehensive and convenient approach. We did not perform any formal economic modelling, but it is worth noting that the Carter report identified a $63 \%$ variation within the $95 \%$ confidence intervals for sickness absence rates across trusts in the English NHS in 2014-15. ${ }^{20}$ The report also estimated that a $1 \%$ improvement in sickness absence rate equates to a $£ 280 \mathrm{~m}$ saving in staff costs - without accounting for lower dependence on agency staff and reduced cancellations. Our data suggest that efforts to improve influenza vaccination uptake by healthcare workers could make a significant contribution to improving this and achieving better value for the NHS. The effect of vaccination on sickness absence was greatest in nurses, the group with the lowest vaccination rates, suggesting that efforts to improve vaccine uptake should focus here.
Finally, influenza vaccination has been shown to improve survival in older populations ${ }^{11}$ and work by the London Respiratory Network has identified influenza vaccination as the highest value intervention for patients with chronic obstructive pulmonary disease (ahead of smoking cessation and pulmonary rehabilitation). ${ }^{21}$ An additional potential benefit of increased healthcare vaccination rates is through modelling of health behaviours to improve patient and carer uptake in influenza vaccination. Vaccinated staff may be more able to encourage patients and carers to themselves have the vaccination.

\section{Conclusion}

These data should encourage HCWs that influenza vaccination is likely to benefit them personally, their colleagues, institution and the NHS.

\section{Conflicts of interest}

The authors have no conflicts of interest to declare.

\section{Author contributions}

NSH, SW, LR, PC and MP conceived the study, MP performed statistical analysis, NSH and MP produced the first draft and all authors contributed to interpretation of the findings and approved the final draft.

\section{Acknowledgements}

This work was supported by the NIHR Respiratory Biomedical Research Unit at Royal Brompton and Harefield NHS Foundation Trust and Imperial College London.

\section{References}

1 England Public Health. Healthcare worker vaccination: clinical evidence (updated August 2016). London: PHE, 2016. http:// www.nhsemployers.org/ /media/Employers/Publications/Flu\%20 Fighter/flu\%20fighter\%20clinical\%20evidence\%20Aug\%202016. pdf [Accessed 11 Augut 2017].

2 Public Health England. Influenza. In: Salisbury D, Ramsay M (eds). Immunisation against infectious disease ('Green Book'), London: PHE, 2015:Chapter 19.

3 Elder AG, O'Donnell B, McCruden EAB et al. Incidence and recall of influenza in a cohort of Glasgow healthcare workers during the 1993-4 epidemic: results of serum testing and questionnaire. BMJ 1996;313:1241-2.

4 Nair H, Holmes A, Rudan I, Car J. Influenza vaccination in healthcare professionals. BMJ 2012;344:e2217.

5 Carman WF, Elder AG, Wallace LA et al. Effects of influenza vaccination of health-care workers on mortality of elderly people in long-term care: a randomised controlled trial. Lancet 2000;355:93-7.

6 Potter J, Stott DJ, Roberts MA et al. Influenza vaccination of health care workers in long-term-care hospitals reduces the mortality of elderly patients. J Infect Dis 1997;175:1-6.

7 Lemaitre M, Meret T, Rothan-Tondeur $\mathrm{M}$ et al. Effect of Influenza vaccination of nursing home staff on mortality of residents: a cluster-randomized trial. J Am Geriatr Soc 2009;57:1580-6.

8 Hayward AC, Harling R, Wetten S et al. Effectiveness of an influenza vaccine programme for care home staff to prevent death, morbidity, and health service use among residents: cluster randomised controlled trial. BMJ 2006;333:1241.

9 Ahmed F, Lindley MC, Allred N et al. Effect of influenza vaccination of healthcare personnel on morbidity and mortality among patients: systematic review and grading of evidence. Clin Infect Dis 2014;58:50-7. 
10 European Centre for Disease Prevention and Control. Vaccine hesitancy among healthcare workers and their patients in Europe - a qualitative study. Stockholm: ECDC, 2015.

11 Nichol KL, Margolis KL, Wouremna J, von Sternberg T. Effectiveness of influenza vaccine in the elderly. Gerontology 1996;42:274-9.

12 Shrikrishna D, Williams S, Restrick L, Hopkinson NS. Influenza vaccination for NHS staff: attitudes and uptake. BMJ Open Respir Res 2015;2:e00079.

13 Smedley J, Poole J, Waclawski E et al. Influenza immunisation: attitudes and beliefs of UK healthcare workers. Occup Environ Med 2007;64:223-7.

14 Caban-Martinez AJ, Lee DJ, Davila EP et al. Sustained low influenza vaccination rates in US healthcare workers. Prev Med 2010;50:210-2.

15 von Elm E, Altman DG, Egger M et al. The Strengthening the Reporting of Observational Studies in Epidemiology (STROBE) statement: guidelines for reporting observational studies. Lancet 2007;370:1453-7.

16 Council of the European Union. Council recommendation 22 December 2009 on seasonal influenza vaccination. Official Journal of the European Union 2009;348:71-2.
17 Saxen H, Virtanen M. Randomized, placebo-controlled double blind study on the efficacy of influenza immunization on absenteeism of health care workers. Pediatr Infect Dis J 1999;18:779-83.

18 Kliner M, Keenan A, Sinclair D et al. Influenza vaccination for healthcare workers in the UK: appraisal of systematic reviews and policy options. BMJ Open 2016;6:e012149.

19 Lewthwaite P, Campion K, Blackburn B et al. Healthcare workers' attitude towards influenza vaccination after the 2009 pandemic. Occup Med 2014;64:348-51.

20 Lord Carter of Coles. Operational productivity and performance in English NHS acute hospitals: unwarranted variations. London: UK Department of Health, 2016.

21 Zoumot Z, Jordan S, Hopkinson NS. Emphysema: time to say farewell to therapeutic nihilism. Thorax 2014;69:973-5.

Address for correspondence: Dr NS Hopkinson, Royal Brompton Hospital, Fulham Rd, London SW3 6NP, UK. Email: n.hopkinson@ic.ac.uk

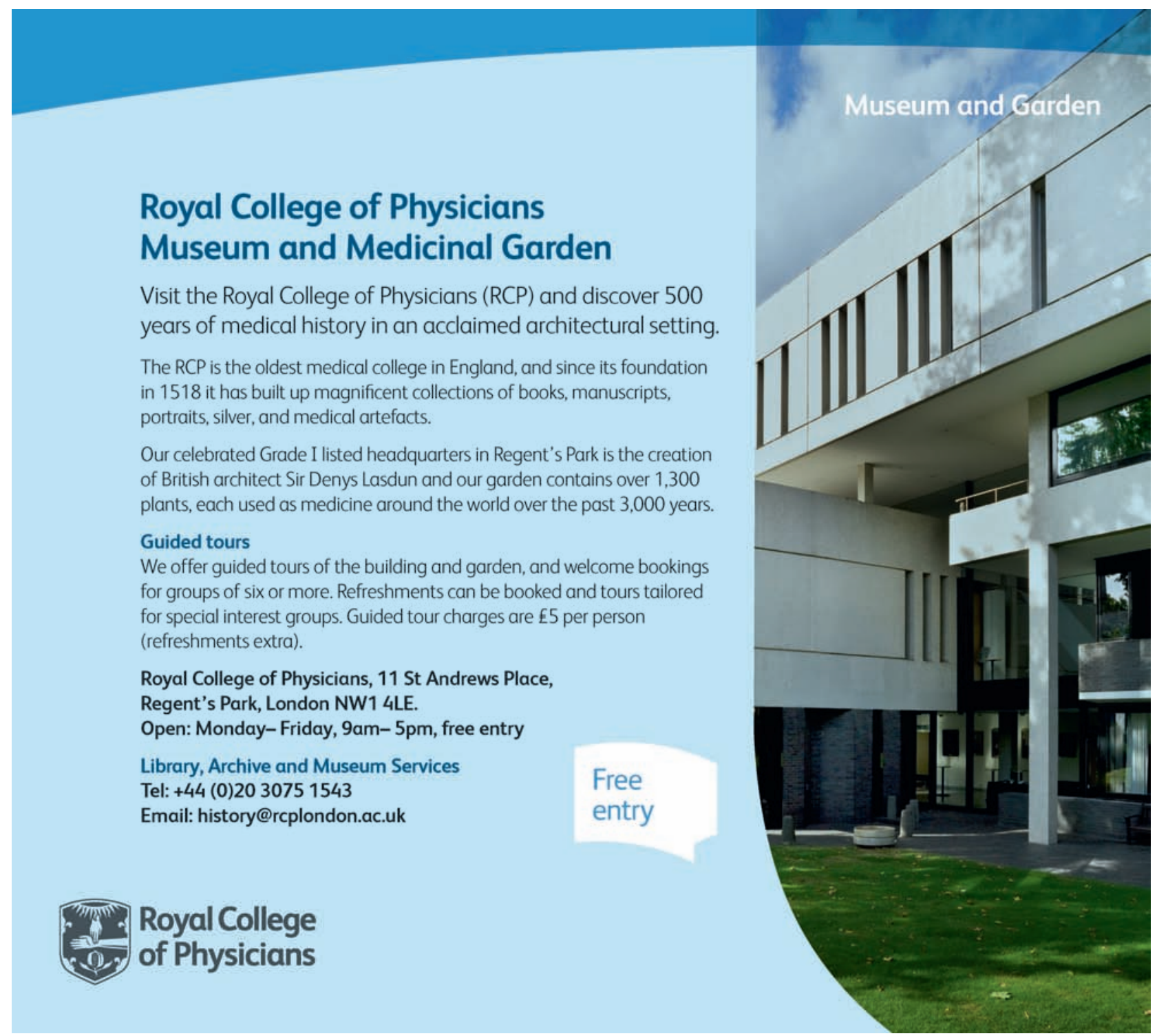

